\title{
The Effects of Electrical Stimulation Mediated by Conductive Polymer on Hypothalamic Neurons as a Potential Model of Schizophrenia
}

\author{
Siti N. Abdul Rahim ${ }^{1,2}$, Xu-Feng Huang ${ }^{2,3}$, Jeremy M. Crook ${ }^{1,2,4}$ \\ ${ }^{1}$ ARC Centre of Excellence for Electromaterials Science, Intelligent Polymer Research Institute \\ University of Wollongong, NSW 2519, Australia \\ snar683@uowmail.edu.au \\ ${ }^{2}$ Illawarra Health and Medical Research Institute, University of Wollongong \\ NSW 2522, Australia \\ ${ }^{3}$ Schizophrenia Research Institute, NeuRA \\ Barker Street Randwick, Sydney NSW 2031, Australia \\ xhuang@uow.edu.au \\ ${ }^{4}$ Department of Surgery, St Vincent's Hospital, the University of Melbourne \\ VIC 3065, Australia \\ jcrook@uow.edu.au
}

\section{Extended Abstract}

Polypyrrole (Ppy) is one of the most extensively studied conductive polymers (CPs) due to its ease of synthesis and surface modifications, high conductivity and its biocompatibility with various cell types [1]. Furthermore, the unique mechanical, electrical and chemical properties of CPs, which may be further enhanced with dopants, make these materials attractive for biomedical applications, such as tissue engineering, drug delivery and biosensors [2][3].

The past decade has shown a resurgence of interest in electrical stimulation therapies, such as deep brain stimulation and transcranial direct current stimulation, for the treatment of schizophrenia (Sz). Sz is a debilitating, neurodevelopmental psychiatric disorder, affecting $\sim 1 \%$ of the population worldwide, with high morbidity rate and no curative treatment for the Sz-induced cognitive deficits to date [4].

One of the key pathologies of $\mathrm{Sz}$ are neural abnormalities, such as reduced neurite outgrowth in the brain [5]. Studies have shown that electrical stimulation, mediated by doped Ppy, promoted nerve cell (PC12) differentiation and increased neurite length and branching of mice primary neurons and human neural stem cells [6][7][8]. Despite published effects of electrical stimulation, the comprehensive mechanism of electrical stimulation at the molecular level is yet to be elucidated. Furthermore, there are insufficient in vitro studies of electrical stimulation focused on the hypothalamus, a brain area affected by Sz. Therefore, this study aims to investigate the molecular effects of electrical stimulation, mediated by Ppy, on hypothalamic neurons and a hypothalamic model of Sz, in addition to the biocompatibility and efficacy of Ppy-mediated electrical stimulation.

Polypyrrole/dodecylbenzene sulfonic acid (Ppy/DBS) films were prepared by galvanostatic electropolymerisation of aqueous pyrrole solution $(0.2 \mathrm{M})$, using an eDAQ potentiostat at a current density of $0.25 \mathrm{~mA} / \mathrm{cm}^{2}$ for 2 minutes with DBS $(0.05 \mathrm{M})$ as the dopant. A standard three-electrode electrochemical set-up, with gold-coated mylar as the working electrode, $\mathrm{Ag} \mid \mathrm{AgCl}$ as the reference electrode and platinum wire mesh as the counter electrode; was used for the polymer growth. Four-well chambers were then glued onto individual Ppy/DBS film strips, and sterilized with 70\% ethanol. Hypothalamic cells (mHypoA-59) were seeded into the chambers at a density of $0.5 \times 10^{4}$ cells/well in DMEM, with $10 \%$ foetal bovine serum and 1\% Penicillin-Streptomycin. Lids comprising platinum mesh electrodes were placed on top of the chambers with the electrodes touching the media. After 24 hours, the cells were electrically stimulated for 3 days using previously established parameters [7]. On day 4, the samples were fixed or collected for assay-ology. Qualitative morphological analysis with immunocytochemistry showed increased neurite outgrowth of the electrically stimulated (ES)-cells compared to the non-ES controls. Quantitative real-time polymerase chain reaction (qRT-PCR) showed electrical stimulation significantly increased the expressions of neuronal gene markers (MAP2 and Dlg4). Future analyses will include additional protein expression assays and quantitative analysis of immunostained cells. The hypothalamic Sz model will also be 
developed by treating the cells with phencyclidine (PCP), a drug known to induce psychosis in humans, which has been used as a pharmacological model of Sz [9]. Preliminary experiments have shown a reduction in the gene expressions of MAP2 and Dlg4 in the PCP-treated cells compared to non-PCP-treated controls. The PCP-treated cells will then undergo electrical stimulation to investigate its efficacy in ameliorating the deficits induced by PCP.

Overall, the study will report on the molecular effects of Ppy/DBS-mediated electrical stimulation on hypothalamic neurons and the hypothalamic PCP-model of Sz, along with the biocompatibility of Ppy/DBS and hypothalamic neuronspolymer interactions.

\section{References}

[1] C. R. Broda, J. Y. Lee, S. Sirivisoot, C. E. Schmidt and B. S. Harrison, "A chemically polymerized electrically conducting composite of polypyrrole nanoparticles and polyurethane for tissue engineering," J Biomed Mater Res A., vol. 98, no. 4, pp. 509-516, 2015.

[2] _-G. Kaur, R. Adhikari, P. Cass, M. Brown and P. Gunatillake, "Electrically conductive polymers and composites for biomedical applications," RSC Adv., vol. 5, pp. 37553-37567, 2015.

[3] _-R. Balint, N. J. Cassidy and S. H. Cartmell, "Conductive polymers: towards a smart biomaterial for tissue engineering," Acta Biomater., vol. 10, no. 6, pp. 2341-2353, 2014.

[4] Ifteni, C. U. Correll, J. M. Kane and P. Manu, "Sudden unexpected death in schizophrenia: an autopsy findings in psychiatric inpatients," Schizophrenia Res., vol. 155, no. 1-3, pp. 72 76, 2014.K. R. Patel, J. Cherian, K. Gohil and D. Atkinson, "Schizophrenia: Overview and Treatment Options," $P \& T$, vol. 39, no. 9, pp. 638-645.

[5] _ Q. Zhang, Y. Yu and X. F. Huang, "Olanzapine prevents the PCP-induced reduction in the neurite outgrowth of prefrontal cortical neurons via NRG1," Sci. Rep., vol. 6, pp. 19581-19591, 2016.

[6] X. Liu, K. J. Gilmore, S. E. Moulton and G. G. Wallace, "Electrical stimulation promotes nerve cell differentiation on polypyrrole/poly(2-methoxy-5aniline sulfonic acid) composites," J Neural Eng., vol. 6, no. 6, pp. 1741-2560, 2009.

[7] E. M. Stewart, N. R. Kobayashi, M. J. Higgins, A. F. Quigley, S. Jamali, S. E. Moulton, R. M. I. Kapsa, G. G. Wallace and J. M. Crook, "Electrical stimulation using conductive polymer polypyrrole promotes differentiation of human neural stem cells: a biocompatible platform for translational neural tissue engineering," Tissue Eng Part C Methods., vol. 21, no. 4, pp. 385-393, 2014.

[8] Q. Zhang, D. Esrafilzadeh, J. M. Crook, R. Kapsa, E. M. Stewart, E. Tomaskovic-Crook, G. G. Wallace and X. F. Huang, "Electrical stimulation using conductive polymer polypyrrole counters reduced neurite outgrowth of primary prefrontal cortical neurons from NRG1-KO and DISC1-LI Mice," Sci. Rep., vol. 7, pp. 42525-42532, 2017.

[9] J. Frohlich and J. D. V. Horn, "Reviewing the ketamine model for schizophrenia," J Psychopharmacol., vol. 28, no. 4, pp. 287-302, 2014. 